\title{
Rapid decline in penicillinase-producing Neisseria gonorrhoeae in Hong Kong associated with emerging 4-fluoroquinolone resistance
}

\author{
K M Kam, K K Lo, Ng K Y Ho, M M Cheung
}

\begin{abstract}
Objective-To study the changes in penicillinase-producing (PPNG) and high-level tetracycline resistant (TRNG) Neisseria gonorrhoeae isolated in Hong Kong associated with emerging quinolone resistance (QRNG) over a two year period from November 1992 to October 1994.

Materials and methods-Four thousand and eighty-six strains of Neisseria gonorrhoeae isolated, of which 432 were PPNG, were examined for susceptibilities to penicillin and tetracycline by an agar dilution method using the breakpoint minimum inhibitory concentrations (MICs) of 1 and $10 \mathrm{mg} / 1$ respectively. Ofloxacin susceptibility was done using 0.1 and $1 \mathrm{mg} / \mathrm{l}$. Penicillinase production was detected by performing the chromogenic cephalosporin nitrocefin test on all penicillin resistant (MIC > $1 \mathrm{mg} / \mathrm{l}$ ) strains.
\end{abstract}

Results-Three thousand and eighty $(75 \cdot 4 \%)$ and $79(1.9 \%)$ strains were found to be penicillin resistant and TRNG (MIC $>10 \mathrm{mg} / \mathrm{l})$ respectively. Sixty-nine strains $(1.7 \%)$ were resistant to both, of which 54 (1.3\%) were PPNG. Three strains were multiply-resistant to penicillin, tetracycline and ofloxacin; however, none was PPNG. While the percentage of penicillin resistant strains remained stable (mean $75 \cdot 5 \%$, SD 7.0), TRNG decreased from $4 \cdot 5 \%$ to $2 \cdot 1 \%$. The most dramatic change was the sharp decline of PPNG from $25.5 \%$ in January 1993 to $4 \cdot 3 \%$ in October 1994 , concurrent with a linear increase in strains with ofloxacin $\mathrm{MIC}>0.1 \mathrm{mg} / 1$. Significant clinical failure was seen in strains having ofloxacin MIC $>1 \mathrm{mg} / 1$ (QRNG), which increased drastically from $0.5 \%$ to $10.4 \%$ during the study period. Selection against PPNG and TRNG strains appeared to occur only when fully quinolone-susceptible strains first become less susceptible (MIC $>0.1$ mg/1), but not when these less susceptible strains become fully resistant (MIC > 1 mg/1).

Conclusion-PPNG is now no longer hyperendemic in Hong Kong. Emergence of QRNG is associated with rapid decline of both PPNG and TRNG. This is the first report of plasmid-curing effect of the 4-fluoroquinolones occurring on an ecological scale.

(Genitourin Med 1995;71:141-144)
Keywords: Neisseria gonorrhoeae; penicillin resistance; 4-fluoroquinolone

\section{Introduction}

Since the first isolation of penicillinase-producing Neisseria gonorrhoeae (PPNG) in $1976,{ }^{12}$ spread of this plasmid-mediated resistance have been documented worldwide. ${ }^{3-5}$ In the South-east Asian region, rates as high as $60-70 \%$ have been documented. ${ }^{67}$ Early reports in Hong Kong have shown that the prevalence of PPNG fluctuated between $31.0 \%$ and $45.2 \%$ in the ten years $1981-90 .{ }^{8}$ Since 1985, single doses of 600 and $400 \mathrm{mg}$ ofloxacin have been used in the Government Social Hygiene (sexually transmitted diseases) Clinics as first-line empirical treatment of urethritis in females and males respectively. In our previous study, we documented the gradual decrease in susceptibility to ofloxacin using three breakpoint concentrations. ${ }^{9}$ We report here the changes in prevalence of PPNG as well as high-level tetracycline resistant Neisseria gonorrhoeae (TRNG) associated with emergence of quinolone-resistance (QRNG) in Hong Kong over a 2-year period.

\section{Materials and methods}

Bacterial strains

The Government Social Hygiene Clinics are distributed over most districts in Hong Kong and mainly serve the lower to middle social class population. A total of 4086 consecutive strains of Neisseria gonorrhoeae, of which 432 were PPNG, isolated from male and female patients attending these clinics between 1 November 1992 and 31 October 1994 were tested for their susceptibilities to penicillin, tetracycline and ofloxacin as well as their beta-lactamase production. Isolation and identification procedures were performed as previously described. ${ }^{9}$

\section{Antibiotic susceptibility tests}

The agar dilution method was used to determine the breakpoint susceptibilities to antibiotics as detailed elsewhere. ${ }^{9}$ For penicillin and tetracycline, 1 and $10 \mathrm{mg} / 1$ were used to indicate resistance (including both plasmidmediated PPNG and chromosomally resistant CMRNG) and high-level resistance respectively. These were chosen because they correlated with clinical resistance and plasmid-mediated tetracycline resistance respectively. Beta-lactamase production was tested on all penicillin resistant strains using chromogenic cephalosporin nitrocefin
Department of

K K Lo

Correspondence to:

Dr K M Kam.

Accepted for publication 6 March 1995 
Table 1 Penicillin resistant (MIC $>1 \mu \mathrm{g} / \mathrm{ml}$ ) Neisseria gonorrhoeae in Hong Kong

\begin{tabular}{llr}
\hline Year/Month & No isolates & $\begin{array}{r}\text { No Penicillin } \\
\text { resistant }(\%)\end{array}$ \\
\hline 9211 & 177 & $131(74 \cdot 01)$ \\
9212 & 176 & $122(69 \cdot 32)$ \\
9301 & 106 & $72(67 \cdot 92)$ \\
9302 & 171 & $114(66 \cdot 67)$ \\
9303 & 198 & $109(55 \cdot 05)$ \\
9304 & 182 & $138(75 \cdot 82)$ \\
9305 & 186 & $147(79 \cdot 03)$ \\
9306 & 194 & $139(71 \cdot 65)$ \\
9307 & 184 & $149(80 \cdot 98)$ \\
9308 & 176 & $154(87 \cdot 50)$ \\
9309 & 173 & $131(75 \cdot 72)$ \\
9310 & 169 & $129(76 \cdot 33)$ \\
9311 & 152 & $116(76 \cdot 32)$ \\
9312 & 178 & $135(75 \cdot 84)$ \\
9401 & 151 & $123(81 \cdot 46)$ \\
9402 & 141 & $110(78 \cdot 01)$ \\
9403 & 186 & $138(74 \cdot 19)$ \\
9404 & 144 & $95(65 \cdot 97)$ \\
9405 & 224 & $181(80 \cdot 80)$ \\
9406 & 196 & $156(79 \cdot 59)$ \\
9407 & 167 & $149(89 \cdot 22)$ \\
9408 & 135 & $103(76 \cdot 30)$ \\
9409 & 180 & $131(72 \cdot 78)$ \\
9410 & 140 & $114(81 \cdot 43)$ \\
\hline
\end{tabular}

(Oxoid, Basingstoke, England). Ofloxacin susceptibility was performed on all strains using 0.1 and $1 \mathrm{mg} / \mathrm{l}$, as the former concentration was the most sensitive indicator of decreasing susceptibility ${ }^{9}$ while growth in the latter (MIC greater than or equal to $2 \mathrm{mg} / \mathrm{l}$ ) correlated with clinical resistance (QRNG).

Control strains that were used in testing of all isolates included our previous panel as well as World Health Organisation Neisseria gonorrhoeae reference strains A-E kindly supplied by Dr John Tapsall (Prince of Wales Hospital, Sydney, Australia). Their MICs were within acceptable range during the study period.

\section{Statistical analysis}

Antibiotic susceptibilities generated in the study were analysed using the SPSS computer package (SPSS/PC + software, v2.0, SPSS Inc.) to obtain best-fit linear regression and correlations. Odds ratios (ORs) with 95\% confidence limits and Fisher exact tests were used to assess the significance of associations and possible relationships between different antibiotics.

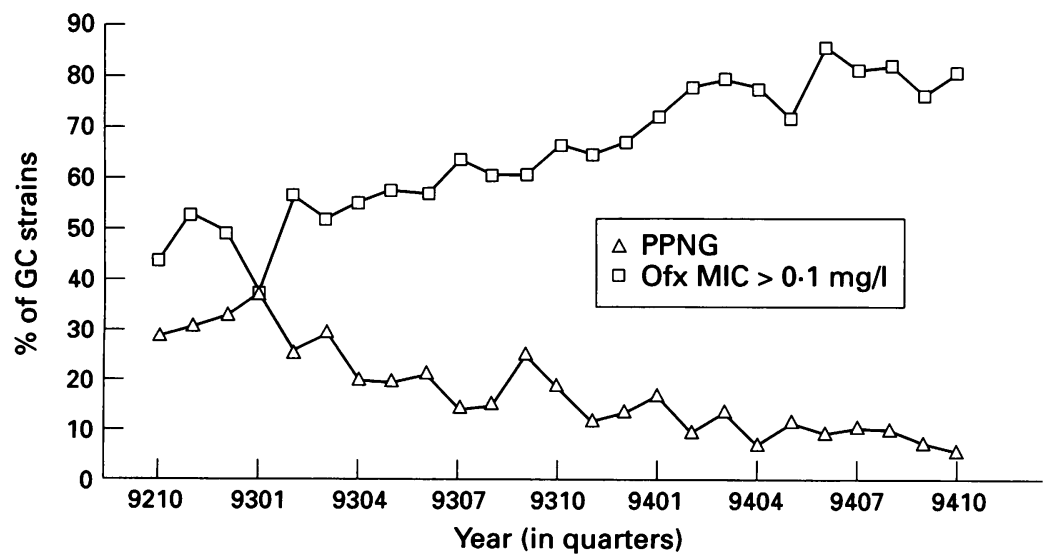

Figure 1 N gonorrhoeae in Hong Kong Nov 1992-Oct 1994. Changes in percentage of penicillin resistant strains that were PPNG, and of strains less susceptible to ofloxacin.

\section{Results}

During the 24 month period, a total of 4086 strains of Neisseria gonorrhoeae were isolated. Table 1 shows the number of positive isolates per month displayed some fluctuations between different months of the year (mean 170, SD 24.6). The percentage of penicillin resistant (MIC >1 mg/l) strains remained quite stable during the study period (mean $75 \cdot 5 \%$, SD $7 \cdot 0$ ).

Figure 1 shows the changes in percentage of penicillin-resistant strains which were PPNG and the proportion of strains resistant to $0.1 \mathrm{mg} / 1$ ofloxacin. It can be seen that the almost linear decreasing susceptibility to ofloxacin observed previously in 1990-92 has continued unabated in the territory throughout the study period. By June 1994, $85 \cdot 2 \%$ of strains showed such decreased susceptibility to ofloxacin. On the other hand, there was a dramatic decline in PPNG from $25.5 \%$ of all isolates in January 1993 to $4.3 \%$ in October 1994, that is, $37 \cdot 5 \%$ and $5.3 \%$ of penicillinresistant (MIC >1 mg/l) strains respectively. Proportionately, the percentage of CMRNG strains had increased. Concomitant with this phenomenon was the disquietingly rapid emergence and spread of QRNG from $0 \%$ in mid-1992, as we documented previously, ${ }^{9}$ to $10.4 \%$ in August 1994 (fig 2). Although there was a brief period of decline towards the end of 1993, the whole year in 1994 showed clear signs of rapid dissemination of these resistant strains. Significant clinical failures in the treatment of uncomplicated urethritis in both males and females were frequently seen in isolates with ofloxacin MIC greater than or equal to $2 \mathrm{mg} / \mathrm{l}$.

The changes in TRNG are shown in fig 3. Our previous observations (unpublished data) indicated that TRNGs were present at low levels in the indigenous gonococcal population. In the present study, there was a drop of TRNG from $4.5 \%$ in December 1992 to $2 \cdot 1 \%$ by the end of the period.

Sixty-nine strains $(1 \cdot 7 \%)$ were resistant to both penicillin (MIC > $1 \mathrm{mg} / \mathrm{l}$ ) and tetracycline (MIC $>10 \mathrm{mg} / \mathrm{l})$, of which $54(1 \cdot 3 \%)$ were PPNG. Three strains were isolated that displayed multiple resistance to penicillin, tetracycline, and ofloxacin; however, none of these were penicillinase producing. Studies are underway to confirm and type these multiple-resistant strains.

Further results of analysis of the degree of inhibition of the 432 PPNG strains in varying concentrations of ofloxacin are shown in table 2 . It can be seen that while there was a definite dose-response relationship between the degree of inhibition and increasing concentrations of ofloxacin, $2 \cdot 4 \%$ of the PPNG strains

Table 2 Distribution of penicillinase-producing Neisseria gonorrhoeae strains for increasing concentrations of ofloxacin

\begin{tabular}{lrrccc}
\hline $\begin{array}{l}\text { Ofloxacin conc. } \\
(\mathrm{mg} / \mathrm{l})\end{array}$ & 0.01 & 0.1 & 0.25 & 1 & 2 \\
$\%$ inhibited & 14.5 & 81.5 & 86.3 & 95.4 & 97.6 \\
\hline
\end{tabular}




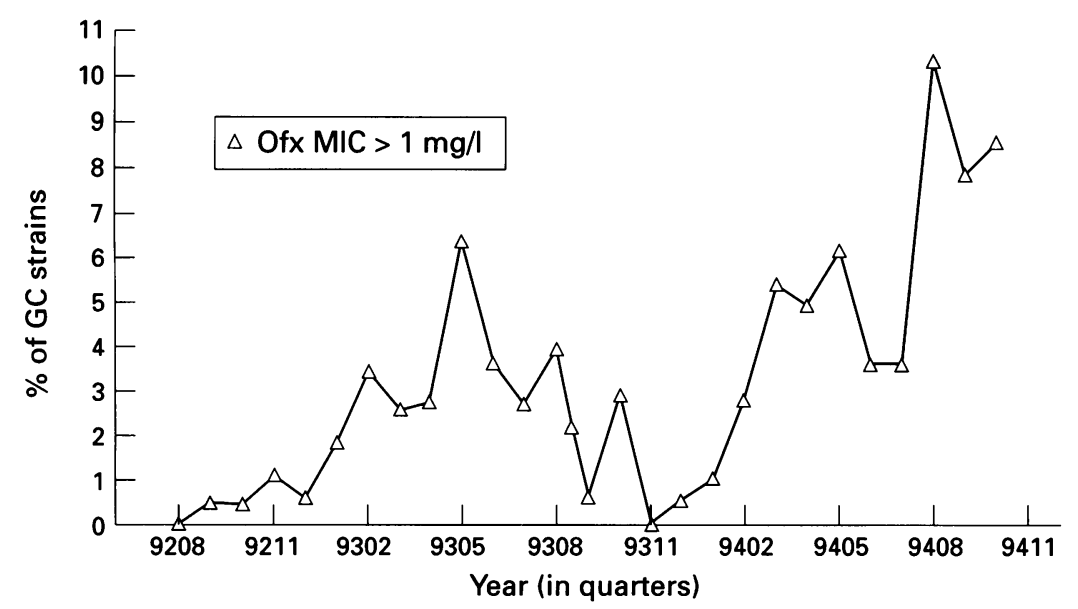

Figure $2 N$ gonorrhoeae in Hong Kong Nov 1992-Oct 1994. The percentage of QRNG strains.

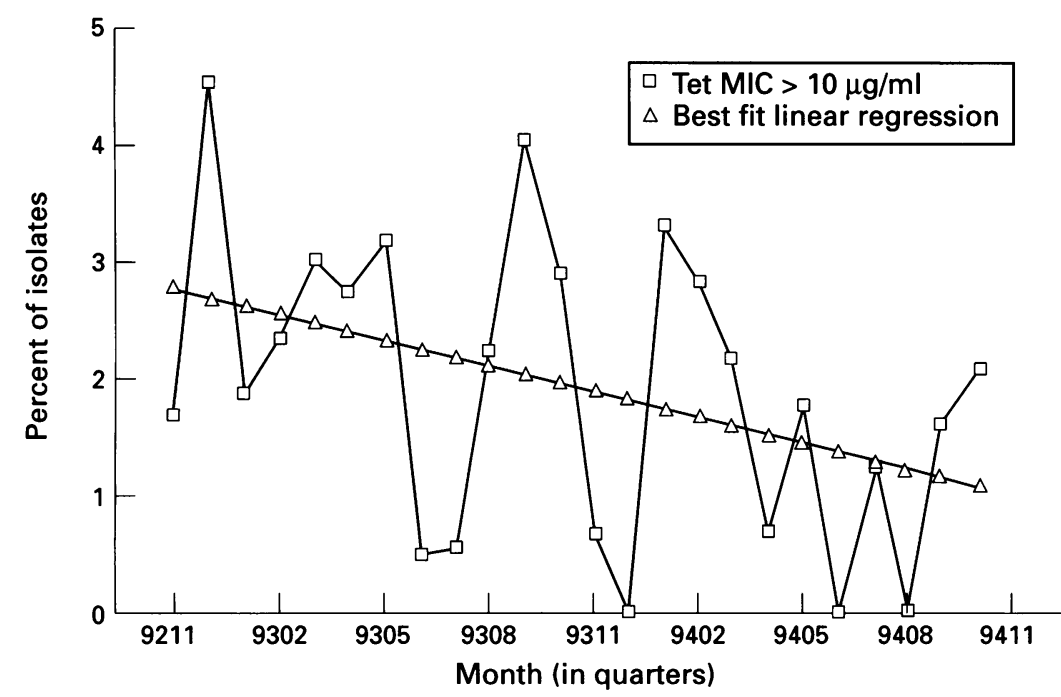

Figure $3 N$ gonorrhoeae in Hong Kong Nov 1992-Oct 1994. The percentage of TRNG.

tested grew even at $2 \mathrm{mg} / \mathrm{l}$ (MIC greater than or equal to $4 \mathrm{mg} / \mathrm{l}$ ).

Out of 149 QRNG strains tested, 122 $(81.9 \%), 20(13.4 \%)$ and $3(2.0 \%)$ were penicillin resistant, PPNG and TRNG respectively. There was a negative association between strains showing decreased quinolone susceptibility (MIC $>0 \cdot 1 \mathrm{mg} / \mathrm{l}$ ) and PPNG [Odds ratio (OR) $0.08,95 \% \mathrm{CI} 0.07$ to 0.11 , p $<0.001$ ] or TRNG (OR $0.19,95 \%$ CI 0.11 to $0.33, \mathrm{p}<0.001$ ), but no such relationship was found between QRNG and PPNG (OR $1.09,95 \%$ CI 0.65 to $1.79, \mathrm{p}=0.74)$ or TRNG (OR 1.03, 95\% CI 0.26-3.42, p = $0 \cdot 76)$. Thus, selection against PPNG and TRNG strains appeared to occur only when fully susceptible strains first become less susceptible (MIC $>0.1 \mathrm{mg} / \mathrm{l}$ ) but not when these less susceptible strains become fully resistant (MIC > $1 \mathrm{mg} / \mathrm{l}$ ).

\section{Discussion}

The susceptibility of the gonococci to antibiotics has been well known to change over time in any locality since the introduction of penicillin. Descriptions of PPNG and TRNG, both plasmid-mediated and easily transmissible, have increased the armamentarium of the gonococci against these first-line antibiotics. Extensive and prolonged use of 4-fluoroquinolones have been reported to be associated with decreasing susceptibility by us $^{9}$ as well as in Japan. ${ }^{10}$ Although we tested only ofloxacin, cross resistance amongst the other 4-fluoroquinolones is expected. ${ }^{11}$ In the present study, we documented that the emergence of QRNG was associated with rapid decline of PPNG and TRNG. As far as we know, this is the first report of the plasmidcuring effect of 4-fluoroquinolones occurring on an ecological scale.

Ison et $a l^{12}$ studied PPNG in the United Kingdom and found rates declining from $8.6 \%$ to $6.5 \%$ within a two year period 1983-4 before the introduction of 4-fluoroquinolones. They suggested more effective diagnosis and treatment, better contact tracing, changes in sexual behaviour or subtle biological changes in the gonococci could be reasons for their observations. Previous studies in Hong Kong ${ }^{8}$ have shown that these various factors may well account for some fluctuations in PPNG rates. However, our observation in the present study on the rapid decline of PPNG to the point of virtual elimination and its possible association with emerging 4-fluoroquinolone resistance has never been suggested before.

While it can be argued that this decline of PPNG and emergence of quinolone resistance may be a coincidental phenomenon, the linear changes over time plus the dose-response between the two lead us to think that there is a real causative relationship. Plasmid-curing effects of quinolones have been well demonstrated in early in vitro studies using enterobacteria. ${ }^{1314}$ Platt and Black studied a multi-plasmid clinical isolate in the presence of sub-inhibitory concentrations of ciprofloxacin and found that plasmids were either consistently retained, lost with variable frequencies or eliminated. ${ }^{15}$ They further postulated that when the sampling site was kept constant even different genera could be compared. Further plasmid analysis of local isolates would help to provide further evidence to confirm our observations and whether certain plasmids have disappeared from our indigenous gonococcal population. In particular, the loss of the conjugative plasmid would prevent the maintenance of a high PPNG prevalence. Another possible mechanism is the inhibition of plasmid conjugation by 4-fluoroquinolones demonstrated by in vitro studies. ${ }^{16} 17$

The question of whether withdrawal of penicillin as first-line therapy for urethritis could have contributed to the decline of PPNG remains largely unanswered. While the selection pressure for penicillin resistant strains may appear to have declined, we think this unlikely to be the sole explanation. One reason is that although PPNG has been declining, the proportion of gonococci which showed penicillin resistance has remained stable, as documented in this study. Thus, our 
gonococcal strains have remained penicillin resistant, but only in the chromosomal form. Another reason is that while penicillin has not been used routinely for treatment of urethritis, consumption of penicillins and cephalosporins for other infectious diseases in the local population has actually been increasing. Also, we did not observe any trend of significant increase in penicillin-sensitive strains in our study, which is what one would expect when penicillin was withdrawn from use as first-line therapy.

In 1993, a total of 82.2 million passengers travelled in and out of Hong Kong including 52.5 million moving to and from China. This massive flux of population means that our gonococcal populations are in constant exchange with our neighbouring countries including Thailand, Korea, the Phillippines, Taiwan and Macau. ${ }^{8}$ Our preliminary observations from contact tracing histories showed that at least $30 \%$ of our patients had a history of contact outside Hong Kong. It is very possible that in places where 4-fluoroquinolones have been used, especially in subinhibitory doses, that the same phenomenon of rapid decline of PPNG and TRNG would be seen. It would be of interest to see if these are also occurring in countries of the South-east Asian region. Further subtyping studies are underway to investigate the effect of emergence of quinolone resistance on possible shifts in our gonococcal populations.

An obvious question is whether there will be eventual elimination of PPNG and TRNG with further selection on exposure of the gonococci to 4-fluoroquinolones. Our observation that $2 \cdot 4 \%$ of our PPNG tested had an ofloxacin MIC at or above $4 \mathrm{mg} / \mathrm{l}$ could have the implication that it would be some time before total elimination could happen. A source of relief was the absence of any strain with multiple resistance to penicillin, tetracycline and ofloxacin in the present study. However, in view of the history of the evolution of gonococcal resistance to antibiotics, there is no reason for complacence and close monitoring is now mandatory for possible emergence of these multiple-resistant strains.

Treatment of these QRNG strains which now comprised $10 \%$ of our strains meant that 4-fluoroquinolones may now be used with much diminished success compared with the early years of introduction. ${ }^{18}$ Although ceftriaxone susceptibility has been reported to be diminished in CMRNG strains, ${ }^{19}$ we are still investigating its clinical usefulness against our QRNG strains.

The authors are grateful to the technical staff in the Clinical Bacteriology Laboratory and Public Health Laboratory at Sa Ying Pun Polyclinic for their expert technical support, and the Director of Health, Dr Margaret Chan, for her permission to publish this report.

1 Phillips I. Beta-lactamase-producing, penicillin-resistan gonococcus. Lancet 1976;2:656-7.

2 Ashford WA, Golash RG, Hemming VG. Penicillinase producing Neisseria gonorrhoeae. Lancet 1976;2:657-8.

3 Zenilman JM, Schwarcz SK, Knapp JS, the Gonococcal Isolate Surveillance Project. National surveillance of antimicrobial resistance in Neisseria gonorrhoeae [abstract no 1155]. In: Program and abstracts of the 28th Interscience Conference on Antimicrobial Agents and Chemotherapy. Washington, DC: American Society for Microbiology, 1988.

4 Dillon JR, Pauze M, Yeung K-H. Molecular and epidemiological analysis of penicillinase-producing strains of Neisseria gonorrhoeae isolated in Canada 1976-84: evoluNeisseria gonorrhoeae isolated in Canada 1976-84: evolu-
tion of new auxotypes and beta-lactamase encoding plastion of new auxotypes and beta-lactam

5 Escamilla J, Bourgeois AL, Gardner CH, Kilpatrick ME. Penicillinase-producing Neisseria gonorrhoeae in various seaport cities of Latin America. Sex Trans Dis 1988;15: $141-3$.

6 Hook EW III, Holmes KK. Gonococcal infections. Ann Int Med 1985;102:229-43.

7 Chu ML, Ho LJ, Lin HC, Wu YC. Epidemiology of Penicillin-resistant Neisseria gonorrhoeae isolated in Taiwan, 1960-1990. Clin Infect Dis 1992:14:450-7.

8 Kam KM, Lai CF, Egglestone S, Chan CBB. Patterns of antibiotic susceptibility of gonococci isolated in Hong antibiotic susceptibility of gonococci isolated in

9 Kam KM, Lo KK, Lai CF, Lee YS, Chan CB. Ofloxacin susceptibilities of 5,667 Neisseria gonorrhoeae strains isolated in Hong Kong. Antimicrob Agents Chemother 1993; 37:2007-8

10 Tanaka $M$, Kumazawa J, Matsumoto T, Kobayashi I High prevalence of Neisseria gonorrhoeae strains with reduced susceptibility to fluoroquinolones in Japan. Genitourin Med 1994;70:90-3

11 Barry AL, Jones RN. Cross-resistance among cinoxacin ciprofloxacin, DJ-6783, enoxacin, nalidixic acid, norfloxacin, and oxolinic acid after in vitro selection of resistant populations. Antimicrob Agents Chemother 1984;25 tant popu.

12 Ison CA, Gedney J, Harris JRW, Easmon CSF Penicillinase producing gonococci: asmen force? Penicillinase producing gono
Genitourin Med 1986;62:302-7.

13 Crumplin GC, Smith JT. The effect of R-factor plasmids on host-cell responses to nalidixic acid. I. Increased susceptibility of nalidixic acid-sensitive hosts. $\mathcal{F}$ Antimicro Chemother 1981;7:379-88.

14 Weisser J, Wiedemann B. Elimination of plasmids by new 4-quinolones. Antimicrob Agents Chemother 1985; 28:700-2.

15 Platt DJ, Black AC. Plasmid ecology and the elimination of plasmids by 4-quinolones. F Antimicrob Chemother 1987;20:137-8

16 Burman LG. R-plasmid transfer and its response to nalidixic acid. $\mathcal{F}$ Bacteriol 1977;131:76-81.

17 Nakamura S, Inoue S, Simizu $M$, Iyobe S, Mitsuhashi S. Inhibition of conjugal transfer of $\mathbf{R}$ plasmids by pipemidic acid and related compounds. Antimicrob A gents Chemother 1976;10:779-85.

18 Dallabetta GA, Hook EW III. Treatment of sexually transmitted diseases with quinolone antimicrobial agents. In Wolfson JS, Hooper DC (ed.) Quinolone Antimicrobial Agents. Washington, DC: American Society for Microbiology 1989

19 World Health Organization. STD Treatment Strategies. Geneva, World Health Organization 1989; WHO/VDT/84.447. 